\title{
Social Network Modelling for Counter Extremism Comparing Criminality in Two Activist Networks
}

\author{
Rosemary Penny ${ }^{1}$, Robert Bowles ${ }^{2}$ and Noémie Bouhana ${ }^{1}$ \\ ${ }^{I}$ Department of Security and Crime Science, University College London, Gower Street, London, U.K. \\ ${ }^{2}$ Department of Mathematics, University College London, Gower Street, London, U.K.
}

Keywords: Social Networks, Agent-based Modelling, Criminology.

\begin{abstract}
This paper examines the phenomenon of extreme protest activities in the environmental and animal rights movements, and how their propagation can be simulated through the construction of agent-based models. It uses criminological theory to examine what factors cause a propensity for violent action to spread across social networks, and uses this as the basis for constructing agent-based models of the activist networks. The differences in the results emerging from the models enable inferences to be made regarding which elements in their construction may cause the differences. Modifying the models to explore how these differences in construction affect the outputs from the models enables us to further understand which real-world factors may contribute to differences in the spread of criminality through the social networks of activists.
\end{abstract}

\section{INTRODUCTION}

Social network modelling has undergone a transformation in recent decades. In the 1980s and 1990s political scientists began to realise the benefits of using graph theory to describe social systems (Wasserman and Faust, 1994); (Diani, 2003). These basic models have since grown in sophistication, with social scientists increasingly looking to the physical sciences for inspiration. Advances in computational power have also had an impact, and social scientists are now turning to computer simulation as a viable method for answering questions in their field, especially those involving systems of interacting actors with heterogeneous characteristics, such as might be found in a social network (Townsley and Johnson, 2008). Simulation has a number of advantages for the social scientist: it is cheap, it is fast, it is easy to control the environment, and it allows for an unlimited number of repetitions (ibid).

This paper takes the field of social network modelling further, by combining the techniques of models rooted in disciplines such as kinetic theory with the social theories of criminology. Drawing on both social and physical science theory, this paper reports on the construction of models describing the spread of criminality through two networks of activists. The differences in the construction of the models are explored to see how they affect the outputs from the models. Using the models in this way enables them to be used as a tool that can suggest explanations for the differences in the ways the two groups protest.

\section{BACKGROUND}

\subsection{Network Modelling}

Network modelling has been well used in fields such as fluid mechanics and statistical physics (Aletti et al., 2007); (Benczik et al., 2009), though the techniques used have only recently been applied to social systems. Several theoretical models have been developed which seek to describe social situations, such as opinion formation and voting models ( $\mathrm{Wu}$ and Huberman, 2004); (Lanchier, 2010) which use techniques originating in fields such as discrete kinetic theory.

Broadly speaking there are two ways in which one can model a network: equation-based modelling (EBM) or agent-based modelling (ABM) (Parunak et al., 1998). The key difference between these two methods is in whether one seeks to model the observables or the individuals in the system, with EBMs examining the relationships between the observables and ABMs considering the attributes of 
and interactions between individuals. ABMs have some advantages over EBMs: they can be easier to construct and understand, they have an extra level of validation available (as they can be tested at either the system or individual level), and they make it easy to conduct "what if" experiments (ibid). As this paper examines attributes of individuals and interactions between them, the models developed in this paper are all ABMs.

The aim of this paper is to see whether these models can be constructed to describe real social networks, in such a way that they can be used to answer questions that would be difficult to answer using conventional social science methods. The networks on which this paper is based are networks of political activists; the intention is to determine whether differences in the levels of criminality among protesters for two different causes can be generated by models constructed to describe their social networks, and if so which aspects of the models have caused those differences. This will provide insight into what factors lead to differences in the levels of criminality among groups of people.

\subsection{Activism and Criminality}

The two types of activists analysed in this research are environmentalists and animal rights activists, for the reason that despite many similarities in their origin they display different characteristics in the ways they protest. Research conducted in the mid1990s into the protest activities of environmentalists found that environmental protesters tended towards demonstrative or confrontational actions, with those wishing to go further opting for minor attacks on property (Seel et al., 2000: 41). Animal rights activists in the period had a much greater tendency towards violence (ibid). More recent research conducted in the USA supports this, showing both that the number of attacks by animal rights activists exceeded those of environmentalists, and that the attacks conducted by animal rights activists were more likely to target people (Carson et al., 2012).

There are many factors which influence whether people engage in criminal activity. Recent advances in criminology have articulated the interplay between individual propensity (morality and selfcontrol) and situational characteristics (moral context and criminogenic factors) to explain the occurrence of crime (Situational Action Theory; see Wikstrom et al., 2012). These interactional models have been used successfully to make sense of fragmented findings on the emergence of the propensity for political violence (Bouhana and
Wikstrom, 2011). The present study builds upon these advances in the criminology of political violence, by examining two of the factors in the developmental process through which an individual acquires a criminal propensity. Since the propensity for committing crime is far harder to measure than crime itself, it is a subject which lends itself well to simulation. The factors examined in this study are the cognitive characteristics that make an individual susceptible to moral change (which we shall refer to as their "moral volatility"), and social contact with individuals with higher levels of criminal propensity (which we shall call "social exposure"). For brevity, we shall refer to an individual's propensity to engage in criminal action as their "level of criminality".

Through the construction of separate models describing the social networks of environmental and animal rights activists based on empirical data, any differences in the number of people with high criminal propensity in the models should provide insight into the reasons why these two groups of activists have developed different propensities for violent action, and thus go some way towards explaining the differences in their violent protest behaviour.

\section{METHODOLOGY}

\subsection{Construction of the Models}

The models in this paper contain no assumptions regarding the level of influence individual activists (the agents) have over each other, or the amount of interaction required for an agent's attributes to change. The social network can thus be represented by an undirected unweighted graph with $N$ nodes representing the activists, and links representing acquaintance. This graph can be described using an $N \mathrm{x} N$ transition matrix $A$, where

$$
A=\alpha_{i j}=\left\{\begin{array}{lc}
0 & \begin{array}{c}
\text { for agents } i \text { and } j \\
\text { not adjacent } \\
\text { for agents } i \text { and } j \\
\text { adjacent }
\end{array}
\end{array}\right.
$$

Each agent $i \in\{0, \ldots, N-1\}$ has two associated attributes: their level of criminality $C$, and their moral volatility $M$. All variables are functions of time $t$, where time is a discrete variable with one time-step for these models taken to represent one month. $\triangle M$ is defined by:

$$
\Delta M(i, t)=M(i, t)-M(i, t-1)
$$


SIMULTECH 2013 - 3rd International Conference on Simulation and Modeling Methodologies, Technologies and Applications

with $\Delta C$ similarly defined. We then define the social exposure to criminality of agent $i$ at time $t, S(i, t)$, as:

$$
S(i, t)=\sum_{j=0}^{j=N-1}\left(\alpha_{i j}(t)(C(j, t)-C(i, t))\right)
$$

Note that zero exposure indicates that the agent's associates are (on average) of the same level of criminality as the agent themselves, and that this definition allows for negative values.

\subsection{Initial State of the Network Models}

The first step is to decide on the initial state of the models: specifically the shape of the networks, and the initial distributions of moral volatility and criminality levels among the agents.

\subsubsection{Shape of the Networks}

As interest in network modelling in the social sciences has increased various studies have been done into the shapes of graphs that most accurately describe social systems. Barabási and Albert (1999) proposed that most real networks are such that the probability that a node is connected to $k$ other nodes follows a power-law distribution, with $P(k) \sim k^{-\gamma}$, where $\gamma$ is between 2 and 3. This is known as a scale-free network (Gómez-Gardeňes and Moreno, 2006). The network models in this paper are created using an algorithm devised by Batagelj and Brandes that generates random scale free networks (2005: 4).

\subsubsection{Distributions of Moral Volatility and Criminality Levels}

For the purposes of these simple models we assume a normal distribution of moral volatility across activists at the start of the simulations; this assumption is supported by data from Wikström's PADS+ cohort study of criminal behaviour among adolescents (2009: 258). To facilitate calculations, $M(i, t)$ shall be a bounded function taking values between 0 and 30, and with a mean of 15 . We shall set the initial moral volatility level for agent $i$ to be a random variable with $M(i, 0) \sim N(15,7.5)$.

For the criminality levels we must determine whether a relationship exists between this variable and moral volatility. To establish this the researcher carried out a survey of animal rights and environmental protesters, testing their morality levels using a method devised by Wikström in his work on crime amongst adolescents (2009), and testing their propensities for criminal activity using a scale devised by Moskalenko and McCauley in their work on activism and radicalism (2009). For the animal rights activists there was a statistically significant positive correlation between these two variables, but no such correlation existed for the environmentalists. To facilitate comparison the two models should start with the same mean criminality level, and so for the animal rights model we define $C(i, 0) \sim N(M(i, 0), 7.5)$, and for the environmentalist model we define $C(i, 0) \sim N(15,7.5)$.

There was no evidence to suggest a relationship between either variable and the number of links an activist has to other activists. The distributions of these attributes are thus independent of node degree.

\subsection{Defining the Rules of the Model}

The next step is to define the rules for how $C, M$, and $S$ change with respect to $t$. Changes to any of the three variables may affect the others, and thus there are six hypotheses regarding the relationships between the variables that must be tested to establish whether they hold, to what extent, and whether differences exist between the two groups of activists. The hypothesis testing was carried out using data collected from the aforementioned survey and interviews with activists for both causes; the results are summarised in the following sections.

\subsubsection{Moral Volatility}

Two hypotheses relate to how moral volatility may change after social exposure or a change in criminality level:

H1: Moral volatility increases after social exposure to criminality: $\Delta M(i, t) \propto S(i, t)$.

$\mathrm{H} 2$ : Moral volatility increases after personal increase in criminality: $\Delta M(i, t) \propto \Delta C(i, t)$.

Data from the survey suggested that $M(i, t)$ should increase a small amount after both a change in criminality level and social exposure. However $M(i, t)$ should also reduce gradually with respect to $t$, as evidence suggests that moral reasoning changes with age (Decety et al, 2012). We therefore define:

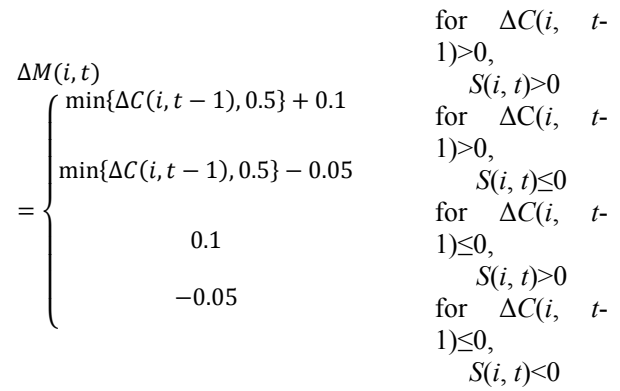

where the term $\min \{\Delta C(i, t-1), 0.5\}$ explains the 
small increase in susceptibility to moral change that comes after an increase in personal criminality, the adding of the constant describes the small effect of social exposure, and the subtracting of the constant describes the effect of age. $M(i, t)$ will also be bounded by 0 and 30 for all $t$.

\subsubsection{Criminality Level}

Another two hypotheses relate to how an individual's criminality level may change based on changes in the other two variables:

H3: An increase in moral volatility leads to an increase in criminality: $\Delta C(i, t) \propto \Delta M(i, t)$.

H4: Level of criminality becomes greater after social exposure to criminality: $\Delta C(i, t) \propto S(i, t)$.

Data from the interviews suggests that a change in criminality level does follow social exposure and can increase rapidly. However criminality level does not appear to change as a result of a change in moral susceptibility. We define:

$$
\begin{aligned}
& \Delta C(i, t) \\
& =\left\{\begin{array}{c}
\frac{S(i, t-1)}{\operatorname{node} \operatorname{degree}(i)} \\
0
\end{array}\right.
\end{aligned}
$$

$$
\begin{gathered}
\text { for } S(i, t-1)>0 \\
\text { otherwise }
\end{gathered}
$$

In addition, as all activists engaged in their protest activities willingly we can say that an activist's moral susceptibility limits their criminality. Thus $C(i, t)$ will have an upper bound, set at $1.5 \times M(i, t)$ for all $t$.

\subsubsection{Social Exposure to Criminality}

The final two hypotheses relate to how social exposure to criminality may change:

H5: Social exposure to criminality increases after personal increase in criminality: $\Delta S(i, t) \propto \Delta C(i, t)$.

H6: Social exposure to criminality increases as moral volatility increases: $\Delta S(i, t) \propto \Delta M(i, t)$

The way a social network changes over time is complicated. Three key issues need to be determined: why people join campaign groups in the first place, how their social circles change once they are in them, and what happens when they leave.

On the first issue, evidence from the interviewees presented a mixed picture: some had migrated from other campaigns, while others had initiated contact with a group when they developed an interest in the cause. The joining of groups will be represented in the models by a proportion of nodes (estimated at $1 \%$ ) forming a new link with another node at each time step. The question is how to determine which other node they form the link with.
Data from the survey carried out by the researcher suggests the most radical people in the animal rights movement were radical beforehand, while those who were not remained peaceful once in the movement. There is no equivalent correlation for environmentalists, from which we can conclude that the extreme environmentalists draw their ranks both from those new to illegal protest activity and from experienced radicals. A possible explanation for this which stems both from the literature and from interviews is that the animal rights movement comprises disparate sub-groups, with the radical groups attracting more radicals to the cause (Jasper and Nelkin, 1992). The environmental groups however join forces during big campaigns, allowing the groups to mingle.

To recreate this effect, the nodes representing animal rights activists will create ties with other nodes of the same criminality level (to simulate the distinct sub-groups), while the nodes representing environmentalists will create ties with other nodes of the same moral volatility (to simulate homophily, the phenomenon of being drawn to those similar to oneself, which is a common feature of social networks (Lanchier, 2010); (Gargiulo and Huet, 2010).

On the second issue of how activists' social circles change after they start campaigning, data from the interviews showed that, with the exception of the most extreme activists, they did not deliberately sever ties with non-activist friends. The most extreme case is incorporated into the model through the severing of ties with activists with a significantly different criminality level. Otherwise, the models will incorporate natural changes in social networks through the random creation of links with friends of friends and the random breaking of links with friends, at a rate which keeps the average number of links per agent relatively static.

The final issue is over how activists leave the network. It is reasonable to suppose that at any time a small number of activists may leave the cause for unknown external reasons. This figure is estimated at $1 \%$ per time-step for both groups.

\subsection{Threats to Model Validity}

There are several threats to the validity of simulation as method. One question is over construct validity: that is whether the model really describes the system it represents (Townsley and Johnson, 2008). A model is in its very essence a simplification of the real world and will never completely describe the system it represents. The question thus becomes 
SIMULTECH 2013 - 3rd International Conference on Simulation and Modeling Methodologies, Technologies and Applications

whether the right simplifications have been made: if variables omitted from the model influence the observables, the model might not be valid. This can be mitigated by testing the outputs of the simulations against real world data. Other threats to the validity of the model are best countered through multiple repetitions of the simulation to check consistency (ibid).

\subsection{Coding the Model}

The models were coded using $\mathrm{C}$ due to its speed and simplicity, enabling the simulation to be run many times with a relatively large number of nodes without requiring any more computer power than that available on a home laptop. The limitations on the computing power available restricted the number of nodes in the models to 1000 . The models were run 100 times each, with $t$ running from 0 to 180 . The simulations thus covered a 15 year period, allowing the model outputs to be compared with empirical data collected by the Association of the British Pharmaceutical Industry and the activist groups themselves dating back to the early 2000s (The Economist, 2011); (Bite Back, 2012).

\section{FINDINGS}

Following the simulations the outputs were compared to assess whether the models produced consistent results. The outputs of interest were the average criminality levels of the agents over time, and the number of criminally-minded agents in the networks (defined to be the number of agents with a criminality level greater than 22). Both models were found to produce consistent results over multiple runs for both output variables of interest. The outputs from the models for the two groups of activists could therefore be compared.

To validate the models, the output for the average criminality levels of the agents was compared with data collected on the number and types of attacks, as discussed in Section 3.5. There are problems with this method of validation, as the number and severity of attacks will not be directly correlated with criminality levels, and the empirical data itself is not entirely reliable. However, comparison of the model outputs with the data does show that the models outputs display a credible extent and rate of change in criminality levels.

In order to determine whether the outputs from the two models are statistically different, the outputs from the 100 simulations were averaged to determine an overall average criminality level and an overall number of criminally-minded agents for each model. These are displayed in Figure 1.

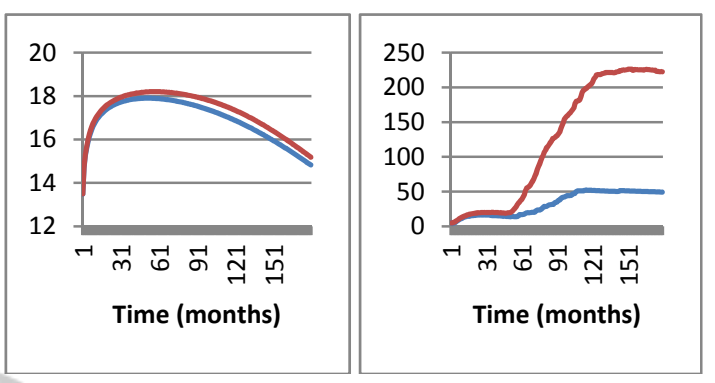

Figure 1: Comparison of the environmentalist (blue) and animal rights (red) models averaged over 100 simulations for average criminality level (left) and number of criminally-minded agents (right).

There is a small but visible difference in the average criminality levels of the two models, with the animal rights model producing a consistently higher criminality level. An independent samples $t$ test confirms that the difference is statistically significant (with the exception of time $t=0$ ), with $p$ values of less than 0.01 .

The difference between the animal rights and environmental models is much more obvious from the diagram showing the difference in the number of criminally-minded agents averaged over 100 simulations, but the variance for this statistic is very large. These data can be better analysed by plotting some examples of the outputs from individual simulations; these are displayed in Figure 2.

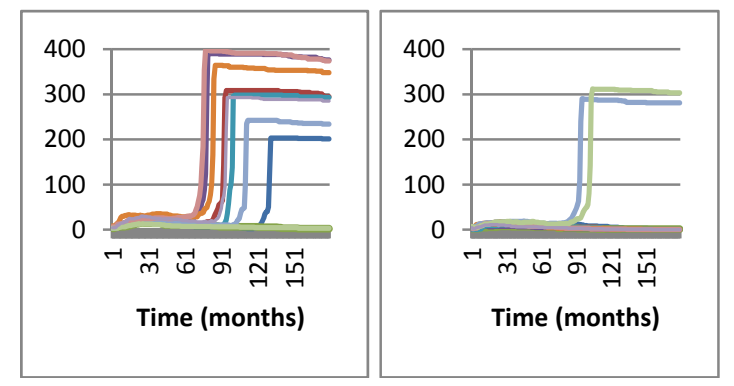

Figure 2: Comparison of the number of criminally-minded agents for 10 simulations for animal rights (left) and environmental (right) activists.

From Figure 2 it can be seen that while there is significant variation across the different runs they follow a similar pattern, with a sharp increase in the number of criminally-minded agents followed by a gentle decline. The timing of the sharp increase, and indeed whether it occurs at all, varies considerably, but it is evident that the increase is more likely to occur in the animal rights model than the 
environmental model.

\subsection{Explaining the Differences}

There are two differences in the construction of the two network models: one difference in the initialisation of the networks, and one difference in the way exposure changes with time. By re-running the simulations and switching the two routines we can learn which of these factors created the differences in output, or if both factors contributed.

After running 100 simulations of each of the model variants it becomes apparent that it is the initialisation of the network that causes the differences in both average criminality level and the numbers of criminally-minded agents in the networks. Therefore it is the correlation between criminality level and moral volatility that causes the increased propensity for criminal activity among animal rights campaigners when compared with environmentalists.

An interpretation of these simulated results could be that where a campaign attracts people whose criminality levels are already closely aligned to their susceptibility to moral change - such as pre-existing activists - then a propensity for violence flourishes among the protesters. This would ultimately lead to a higher likelihood of criminal behaviour taking place during protests attended by these individuals.

\section{FURTHER WORK}

The models are naively constructed, with many assumptions made, and this limits their faithfulness to reality. This becomes evident when the simulations are run for longer and the distribution of criminality levels across the agents is analysed. Figure 3 shows scatterplots illustrating the criminality levels of all 1,000 agents at time $t=0$ and $t=500$ for one typical run of the model.

From Figure 3 it can be seen that at time $t=500$ the criminality levels cluster around zero and a higher value, resulting in a bimodal distribution. Further, examination of the network at time $t=500$ reveals that all links between agents are gone, leaving every agent isolated. While a plausible explanation for this could be argued - for instance that after 500 months (over 40 years) people are unlikely to have the same friends, and will either have lost their propensity for violence altogether or it will be deeply entrenched - a more likely explanation is that this is due to a lack of sophistication in the model.

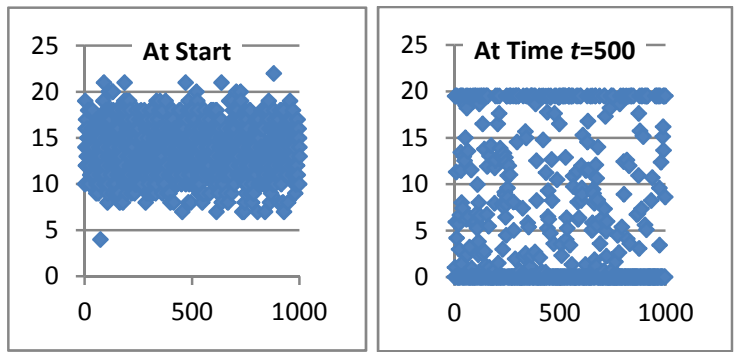

Figure 3: Criminality levels for all agents for one typical run of the model at time $\mathrm{t}=0$ and $\mathrm{t}=500$.

There are a number of ways the models could be improved to make them more faithful to reality. One failing is the lack of new blood introduced into the networks. The graphs showing how average criminality levels change with time demonstrate that the models reach a steady state after a few years, with a gentle tailing off of criminality levels which is almost certainly due to the changes in moral reasoning that come with age. It would be more realistic to introduce a number of new agents with each time step that are initialised in the same way as the rest of the network at time $t=0$, and to entirely remove the old agents that have cut their ties. This amendment would be further improved with empirical data providing realistic figures as to how many people join and leave the movements each year.

Other improvements could be made to the models with sufficient data about the social circles of the activists. The main assumptions made in the construction of the models concern the making and breaking of friendships. It was outside the scope of the research to date to collect detailed data about the way peoples' social circles change over time; a longitudinal study tracking the social links within a specific group of activists would need to be done to make this aspect of the models more realistic.

A further change given such data could be to use a directed weighted graph for the network models instead of an undirected unweighted graph. A directed weighted graph would have the capacity to model the strengths of social ties and how much each individual influences or is influenced by those around them. This could easily be incorporated into the models by changing the values in the transition matrix so that it holds values other than just 0 and 1 .

Finally, these models take into account only two factors that contribute to the development of a propensity for criminal action. For greater realism additional variables should be incorporated, for example the activists' capacity for self-control, and factors contributing to their susceptibility to being 
SIMULTECH 2013 - 3rd International Conference on Simulation and Modeling Methodologies, Technologies and Applications

exposed to criminality, such as place of residence.

With sufficient improvement to these models they could be used to make more reliable judgments regarding which factors influence the levels of criminality in a social network, and the size of the effect those factors have. The simple versions described in this paper are an important first step.

\section{CONCLUSIONS}

This paper has demonstrated that combining social theories such as those found in criminology with network modelling techniques rooted in the physical sciences produces a powerful tool that could be of great use in fields such as research into extremism. The models used here are simplistic and are thus limited in their ability to provide insight into real world phenomena, but they form an important first step. Further empirical data about the social networks of activists and their levels of criminality would allow greater complexity to be incorporated into these basic models to make them truer to life. This increased construct validity would give the models the power to answer with much greater certainty questions such as what critical factors affect the spread of criminality through a social network. These models could then become of practical use to policy makers, both in the field of crime prevention and in other fields where conditions can spread through social interaction.

\section{REFERENCES}

Aletti, G., Naldi, G., Toscani, G. , 2007. First-Order Continuous Models of Opinion Formation. In Journal of Applied Mathematics, 67 (3), 837-853.

Barabási, A.-L., Albert, R., 1999. Emergence of Scaling in Random Networks. In Science, 509-512.

Batagelj, V., Brandes, U., 2005. Efficient generation of large random networks. In Physical Review E, 71 (036113).

Benczik, I. J., Benczik, S. Z., Schmittmann, B., Zia, R. K., 2009. Opinion dynamics on an adaptive random network. In Physical Review E, 79 (046104).

Bite Back, 2012. Diary of Actions. Retrieved July 20, 2012, from Bite Back: http://www.directaction.info/news.htm

Bouhana, N., Wikström, P.-O., 2011. Al Qa'ida-influenced Radicalisation: A Rapid Eviden ce Assessment Guided by Situational Action Theory. Occasional Paper 97, Research, Development and Statistics, Office for Security and Counter-Terrorism. Home Office. London.

Carson, J. V., LaFree, G., Dugan, L., 2012. Terrorist and
Non-Terrorist Criminal Attacks by Radical Environmental and Animal Rights Groups in the United States, 1970-2007. In Terrorism and Political Violence, 24 (2), 295-319.

Decety, J., Michalska, K. J., Kinzler, K. D., 2012. The Contribution of Emotion and Cognition to Moral Sensitivity: A Neurodevelopmental Study. In Cerebral Cortex, 22 (1), 209-220.

Diani, M., 2003. 'Leaders' or 'brokers'? Positions and influence in social movement networks. In M. Diani, \& D. McAdam, Social movements and networks (pp. 105-122). Oxford University Press. Oxford.

Gargiulo, F., Huet, S., 2010. Opinion dynamics in a groupbased society. In EPL, 91 (58004).

Gómez-Gardeňes, J., Moreno, Y., 2006. From Scale-free to Erdos-Rényi Networks. In Physical Review E, 73 (056124).

Jasper, J. M., Nelkin, D., 1992. The Animal Rights Crusade: The Growth of a Moral Protest. The Free Press, Macmillan. New York.

Lanchier, N., 2010. Opinion dynamics with confidence threshold: an alternative to the Axelrod model. In ALEA, 7, 1-18

Moskalenko, S., McCauley, C., 2009. Measuring Political Mobilization: The Distinction Between Activism and Radicalism. In Terrorism and Political Violence, 21 (2), 239-260.

Parunak, H. V., Savit, R., Riolo, R. L., 1998. Agent-Based Modeling vs. Equation-Based Modeling: A Case Study and Users' Guide. In Proceedings of Multi-agent systems and Agent-based Simulation (MABS '98), LNAI 1534

Seel, B., Paterson, M., Doherty, B., 2000. Direct action in British environmentalism. Routledge. London.

The Economist, 2011. Britain: A beastly business; Animal-rights protests. In The Economist , 401 (8756), 42.

Townsley, M., Johnson, S., 2008. The need for systematic replication and tests of validity in simulation. In L. Liu, \& J. Eck, Artificial crime analysis systems: using computer simulations and geographic information systems (pp. 1-18). Information Science Reference. Hershey.

Wasserman, S., Faust, K., 1994. Social Network Analysis: Methods and Applications. Cambridge University Press. Cambridge.

Wikström, P.-O., 2009. Crime propensity, Criminogenic Exposure and Crime Involvement in Early to Mid Adolescence. In Monatsschrift fur Kriminologie und Strafrechtsreform, 92, 523-566.

Wikström, P.-O., Oberwittler, D., Treiber, K., Hardie, B., 2012. Breaking Rules: The Social and Situational Dynamics of Young People's Urban Crime. Oxford University Press. Oxford.

Wu, F., Huberman, B. A., 2004. Social Structure and Opinion Formation. 